\title{
Quantitative Trait Loci Mapping for External Egg Traits in $F_{2}$ Chickens
}

\author{
Tatsuhiko Goto ${ }^{1,2,4}$, Akira Ishikawa ${ }^{2,3}$, Minori Yoshida ${ }^{1}$, Naoki Goto ${ }^{1,5}$, Tetsuya Umino ${ }^{1}$, \\ Masahide Nishibori $^{1,2}$ and Masaoki Tsudzuki ${ }^{1,2}$ \\ ${ }^{1}$ Graduate School of Biosphere Science, Hiroshima University, Higashi-Hiroshima, Hiroshima 739-8528, Japan \\ ${ }^{2}$ Japanese Avian Bioresource Project Research Center, Hiroshima University, Higashi-Hiroshima, Hiroshima 739-8528, Japan \\ ${ }^{3}$ Graduate School of Bioagricultural Sciences, Nagoya University, Chikusa, Nagoya, Aichi 464-8601, Japan \\ ${ }^{4}$ Present address: College of Agriculture, Ibaraki University, Ami, Ibaraki 300-0393, Japan \\ ${ }^{5}$ Present address: Institute de Sélection Animale B.V., 5830 AC Boxmeer, The Netherlands-EU
}

\begin{abstract}
Quantitative trait locus (QTL) mapping of external egg traits in chickens was carried out with 143 microsatellite markers using $388 \mathrm{~F}_{2}$ hens obtained from an intercross of a Japanese Large Game (Oh-Shamo) male and three White Leghorn females. External egg traits, such as the weight and length of eggs and the weight, thickness, strength, and color of eggshells were measured at three different stages of egg production, i.e. early, middle, and late stages. QTL analyses revealed 10 significant and three suggestive QTLs with main effects on external egg traits on chromosomes $1,2,4,5,8,10,11,12,17$, and Z. The QTL alleles derived from Oh-Shamo, one of the indigenous Japanese breeds, uniquely increased most external egg traits, whereas some QTL alleles had opposite effects on phenotypic differences between Oh-Shamo and White Leghorn. The QTLs detected accounted for 3.09-15.39\% of the phenotypic variances. Although the QTLs on chromosomes 5, 8, and Z were found throughout all three stages, the others were identified at only one or two stages. Epistatic QTLs for external egg traits were not detected in the present study. The age-related changes of our main-effect QTLs illustrate one aspect of the complex genetic basis of external egg traits.
\end{abstract}

Key words: age-related change, chicken, external egg quality, mapping, quantitative trait loci

J. Poult. Sci., 51: 118-129, 2014

\section{Introduction}

A great number of researches have been conducted to understand the genetic basis of quantitative traits which are controlled by multiple quantitative trait loci (QTLs), environment, and their interactions (Mackay et al., 2009). It is important to identify the chromosomal regions of QTLs affecting such economically important traits, because such positional information will be useful to improve the livestock breeding performances in the future. In chickens, many QTL mapping studies have detected loci associated with growth, egg, meat, behavior, and disease traits (Abasht et al., 2006; Hu et al., 2007, 2012).

External egg traits, which are directly related to the quality of egg products, can be defined as egg weight, egg size, eggshell color, eggshell strength, eggshell weight, and eggshell thickness (Fulton, 2004; Roberts, 2004). In addition, the structure and formation of the eggshell and the properties of

Received: May 26, 2013, Accepted: August 22, 2013

Released Online Advance Publication: September 25, 2013

Correspondence: Dr. M. Tsudzuki, Graduate School of Biosphere Science,

Hiroshima University, Higashi-Hiroshima 739-8528, Japan.

(E-mail: tsudzuki@hiroshima-u.ac.jp) the eggshell matrix proteins have been investigated (Nys et al., 2004; Hincke et al., 2010). Dunn et al. (2009) reported that DNA polymorphisms in six eggshell-related genes, ovocleidin-116, osteopontin, ovocalyxin-32, ovalbumin, estrogen receptor, and carbonic anhydrase II, were associated with eggshell quality. However, these genes were accounted for only a part of the entire genetic variation of eggshell quality. Accordingly, it is very likely that many QTLs with main and epistatic effects on these traits remain undiscovered.

There have been some reports in which eggshell quality (Roland, 1979) and brown eggshell color (Odabasi et al., 2007) are said to be influenced by age of hens. Hence, agerelated changes of QTLs may have contributed to these egg traits. However, the previous QTL studies on egg traits focused on only one or two stages of egg production. In the present study, we measured external egg traits at three different stages of egg production, i.e. early, middle, and late stages, in an attempt to understand age-related changes of QTL expression.

In the present study, we used the Japanese Large Game (Oh-Shamo) and White Leghorn breeds as parental breeds for development of a QTL mapping population. The Oh- 
Table 1. Microsatellite DNA markers genotyped in the present study

\begin{tabular}{|c|c|c|c|c|c|c|c|}
\hline Marker & $\begin{array}{l}\text { Chromosome } \\
\text { or } \\
\text { linkage } \\
\text { group }\end{array}$ & $\begin{array}{l}\text { Map } \\
\text { position } \\
(\mathrm{cM})^{1}\end{array}$ & $\begin{array}{l}\text { Reference } \\
\text { map } \\
\text { position } \\
(\mathrm{cM})^{2}\end{array}$ & Marker & $\begin{array}{l}\text { Chromosome } \\
\text { or } \\
\text { linkage } \\
\text { group }\end{array}$ & $\begin{array}{l}\text { Map } \\
\text { position } \\
(\mathrm{cM})^{1}\end{array}$ & $\begin{array}{l}\text { Reference } \\
\text { map } \\
\text { position } \\
(\mathrm{cM})^{2}\end{array}$ \\
\hline$M C W 0248$ & 1 & 0 & 19 & $A B R 0017$ & 8 & 0 & 0 \\
\hline LEI0209 & 1 & 24.5 & 56 & $M C W 0305$ & 8 & 27.3 & 15 \\
\hline$M C W 0010$ & 1 & 35.1 & 72 & MCW0095 & 8 & 37.2 & 26 \\
\hline$M C W 0111$ & 1 & 67.6 & 118 & $A D L 0345$ & 8 & 56.2 & 56 \\
\hline ROS0327 & 1 & 70 & 118 & ROS0075 & 8 & 95.9 & 94 \\
\hline$A D L 0188$ & 1 & 79.1 & 133 & ROS0078 & 9 & 0 & 0 \\
\hline$A D L 0124$ & 1 & 84.8 & 143 & $M C W 0329$ & 9 & 42.6 & 54 \\
\hline LEI0068 & 1 & 91.1 & 151 & $M C W 0135$ & 9 & 52.2 & 61 \\
\hline LEI0146 & 1 & 102.5 & 169 & ABR0526 & 9 & 79.2 & $*$ \\
\hline$M C W 0112$ & 1 & 132.4 & 205 & ADL0259 & 9 & 93.5 & 122 \\
\hline$M C W 0058$ & 1 & 157.8 & 241 & $M C W 0134$ & 9 & 104.4 & 132 \\
\hline LEI0101 & 1 & 172.4 & 259 & $A D L 0272$ & 10 & 0 & 44 \\
\hline LEI0198 & 1 & 256.7 & 364 & $A D L 0106$ & 10 & 19.3 & 88 \\
\hline$M C W 0036$ & 1 & 282.3 & 386 & LEI0112 & 10 & 37.5 & 107 \\
\hline LEI0106 & 1 & 319.2 & 426 & $A D L 0123$ & 11 & 0 & 22 \\
\hline$M C W 0102$ & 1 & 336.8 & 441 & $A D L 0210$ & 11 & 22.5 & 54 \\
\hline$A D L 0245$ & 1 & 362.7 & 459 & MCW0066 & 11 & 35.5 & 69 \\
\hline ABR0328 & 1 & 367.5 & 475 & $A B R 0037$ & 11 & 57.5 & 90 \\
\hline GCT0032 & 1 & 407.3 & 518 & $A D L 0372$ & 12 & 0 & 0 \\
\hline MCW0107 & 1 & 460.3 & 565 & $A D L 0044$ & 12 & 61.9 & 44 \\
\hline LEIO124 & 2 & 0 & $*$ & $M C W 0340$ & 13 & 0 & 2 \\
\hline$M C W 0205$ & 2 & 9.1 & 2 & LEI0251 & 13 & 46.9 & 47 \\
\hline MCW0184 & 2 & 73.8 & 60 & $A D L 0214$ & 13 & 79.7 & 70 \\
\hline ADL0190 & 2 & 75.6 & 62 & $M C W 0104$ & 13 & 81.1 & 74 \\
\hline$A D L 0176$ & 2 & 133.6 & 116 & MCW0296 & 14 & 0 & 0 \\
\hline$M C W 0062$ & 2 & 182.5 & 172 & $M C W 0123$ & 14 & 45.2 & 45 \\
\hline LEI0096 & 2 & 230.8 & 233 & $A D L 0263$ & 14 & 68.2 & 72 \\
\hline$M C W 0173$ & 2 & 240.6 & 243 & $A B R 0517$ & 14 & 81.5 & $*$ \\
\hline$M C W 0027$ & 2 & 251.2 & 255 & ADL0206 & 15 & 0 & 0 \\
\hline LEI0147 & 2 & 270.5 & 282 & $M C W 0031$ & 15 & 1.5 & 7 \\
\hline$M C W 0185$ & 2 & 286.8 & 302 & $M C W 0211$ & 15 & 54.8 & 49 \\
\hline LEI0237 & 2 & 302.5 & 320 & ABR0530 & 17 & 0 & $*$ \\
\hline LEI0070 & 2 & 363.8 & 379 & $A D L 0293$ & 17 & 14 & 26 \\
\hline$A D L 0146$ & 2 & 387.6 & 403 & $M C W 0330$ & 17 & 27.7 & 41 \\
\hline$M C W 0261$ & 3 & 0 & 0 & ABR0387 & 17 & 32.2 & $*$ \\
\hline$M C W 083$ & 3 & 97.1 & 51 & $A D L 0202$ & 17 & 46.6 & 70 \\
\hline ADL0229 & 3 & 149.6 & 111 & ABR0374 & 18 & 0 & $*$ \\
\hline LEI0115 & 3 & 179.8 & 143 & MCW0217 & 18 & 16.1 & 24 \\
\hline$M C W 0252$ & 3 & 223.8 & 201 & ABR0133 & 19 & 0 & $*$ \\
\hline LEI0223 & 3 & 246.3 & 226 & ABR0180 & 19 & 24 & $*$ \\
\hline$M C W 0016$ & 3 & 252.3 & 247 & MCW0304 & 19 & 32.7 & $*$ \\
\hline MCW0156 & 3 & 271.3 & 276 & MCW0287 & 19 & 35.7 & 40 \\
\hline$A D L 0143$ & 4 & 0 & 0 & MCW0119 & 20 & 0 & 0 \\
\hline$A D L 0203$ & 4 & 40.7 & 35 & $A D L 0125$ & 20 & 12.5 & 26 \\
\hline LEI0095 & 4 & 87.8 & 78 & $A D L 0262$ & 23 & 0 & 0 \\
\hline$M C W 0005$ & 4 & 105.1 & 101 & MCW0165 & 23 & 1.9 & 1 \\
\hline$A D L 0266$ & 4 & 133.1 & 138 & ROSO302 & 24 & 0 & 20 \\
\hline LEI0076 & 4 & 169.9 & 182 & $M C W 0301$ & 24 & 19.9 & 48 \\
\hline$A B R 0331$ & 4 & 176.5 & 183 & APOA1sts 1 & 24 & 26.8 & 53 \\
\hline$M C W 0240$ & 4 & 201.1 & 201 & APOA1sts 2 & 24 & 27 & 53 \\
\hline$A B R 0622$ & 4 & 233.8 & $*$ & MCW0262 & 26 & 0 & 26 \\
\hline$A B R 0045$ & 4 & 287.2 & $*$ & MCW0069 & 26 & 13.4 & 47 \\
\hline$M C W 0038$ & 5 & 0 & 71 & ABR0006 & 26 & 38.2 & 56 \\
\hline$M C W 0214$ & 5 & 21.8 & 88 & MCW0233 & 27 & 0 & 19 \\
\hline$M C W 0078$ & 5 & 34.5 & 93 & $M C W 0328$ & 27 & 33 & 47 \\
\hline$M C W 0223$ & 5 & 57.9 & 123 & GCT0022 & 27 & 38.9 & 50 \\
\hline
\end{tabular}


Table 1. (Continued)

\begin{tabular}{|c|c|c|c|c|c|c|c|}
\hline Marker & $\begin{array}{l}\text { Chromosome } \\
\text { or } \\
\text { linkage } \\
\text { group }\end{array}$ & $\begin{array}{l}\text { Map } \\
\text { position } \\
(\mathrm{cM})^{1}\end{array}$ & $\begin{array}{l}\text { Reference } \\
\text { map } \\
\text { position } \\
(\mathrm{cM})^{2}\end{array}$ & Marker & $\begin{array}{l}\text { Chromosome } \\
\text { or } \\
\text { linkage } \\
\text { group }\end{array}$ & $\begin{array}{l}\text { Map } \\
\text { position } \\
(\mathrm{cM})^{1}\end{array}$ & $\begin{array}{l}\text { Reference } \\
\text { map } \\
\text { position } \\
(\mathrm{cM})^{2}\end{array}$ \\
\hline$A D L 0233$ & 5 & 77.6 & 151 & LEI0135 & 28 & 0 & 0 \\
\hline$A D L 0166$ & 5 & 91.9 & 162 & ADL0254 & 28 & 20.9 & 25 \\
\hline ROS0330 & 5 & 121.9 & 198 & $A D L 0284$ & 28 & 23.5 & 25 \\
\hline MCW0118 & 6 & 0 & $*$ & ADL0299 & 28 & 55.4 & 60 \\
\hline ABR0028 & 6 & 18.9 & * & ABR0597 & 32 & 0 & $*$ \\
\hline$A D L 0138$ & 6 & 21 & 56 & GCT0004 & E50C23 & 0 & 40 \\
\hline LEI0092 & 6 & 28.8 & 59 & $A D L 0022$ & $\mathrm{Z}$ & 0 & 0 \\
\hline LEI0196 & 6 & 67.4 & 110 & $M C W 0055$ & $\mathrm{Z}$ & 28.4 & 15 \\
\hline$A B R 0041$ & 7 & 0 & $*$ & $M C W 0258$ & $\mathrm{Z}$ & 38.6 & 26 \\
\hline MCW0120 & 7 & 8.4 & 44 & $A D L 0273$ & Z & 56.2 & 73 \\
\hline ABR0397 & 7 & 19.8 & $*$ & $A D L 0201$ & Z & 83 & 87 \\
\hline$M C W 178$ & 7 & 33.3 & 72 & $M C W 0154$ & Z & 97.6 & 95 \\
\hline ABR0195 & 7 & 36.2 & $*$ & LEI0121 & Z & 120 & 131 \\
\hline$M C W 0183$ & 7 & 42.8 & 86 & ABR0119 & Z & 134.7 & $*$ \\
\hline LEI0158 & 7 & 76.4 & 122 & ABR0289 & Z & 148.3 & * \\
\hline$A D L 0169$ & 7 & 105 & 165 & & & & \\
\hline
\end{tabular}

${ }^{1}$ Kosambi genetic distance from the first marker on each chromosome or linkage group.

${ }^{2}$ Reference position shown in ArkDB database by Roslin Bioinfomatics Group (http://www.thearkdb.org/).

* Not located on the consensus linkage map in 2005 (Schmid et al., 2005).

Shamo is one of the most famous Japanese indigenous chicken breeds and it is kept for cock fighting (Tsudzuki, 2003). In contrast, the White Leghorn which originated from Italy has been selected intensely for egg production. Since these breeds have large genetic divergence (Osman et al., 2006), many unique QTLs will be found. This study therefore aims to detect QTLs affecting external egg quality and illustrate age-related changes of the QTLs detected.

\section{Materials and Methods}

\section{Animals}

An Oh-Shamo male was mated to three White Leghorn females to produce $23 \mathrm{~F}_{1}$ individuals. Three hundred and eighty-eight $F_{2}$ females were created by full-sib matings between four $\mathrm{F}_{1}$ males and $19 \mathrm{~F}_{1}$ females. All individuals were reared as described by Tsudzuki et al. (2007). Hens were kept under a photoperiod condition of $14 \mathrm{~h}$ light and 10 $\mathrm{h}$ dark. All the chickens were treated according to the rules described in Standards Relating to the Care and Management of Experimental Animals (Prime Ministers's Office, Japan, 1980) and Guide for the Use of Experimental Animals in Universities (The Ministry of Educations, Science, Sports, and Culture, Japan, 1987).

\section{Phenotypic Measurements}

External egg traits were measured at three different production stages: first egg stage, $300 \mathrm{~d}$ of hen age, and $400 \mathrm{~d}$ of hen age. First 10 eggs laid by hens were measured as data for the first egg stage. Eggs at $300 \mathrm{~d}$ and $400 \mathrm{~d}$ of ages were collected within two weeks from $300 \mathrm{~d}$ and $400 \mathrm{~d}$ of age onwards. External egg traits measured were egg weight (EW), eggshell weight (SW), lengths of the long and short axes of the egg (LLE and LSE), eggshell thickness of the narrow end, blunt end, and equator of the egg (STN, STB, and STE), eggshell strength (SS), and lightness, redness, and yellowness of the eggshell color (SCL, SCR, and SCY). SS was measured at the pole by using an eggshell strength tester (Fujihira Industry, Japan). Eggshell color was measured with a Minolta Chroma Meter CR-300 (Konica Minolta, Japan). In the present study, the first egg stage, $300 \mathrm{~d}$ of age, and $400 \mathrm{~d}$ of age were referred to as the early (E-), middle (M-), and late (L-) production stages, respectively.

\section{Genotyping and Map Construction}

Genomic DNA was extracted from individual blood samples. Fully informative 143 microsatellite markers on 26 autosomes and the $\mathrm{Z}$ chromosome (Table 1) were genotyped following the method of Tadano et al. (2007). A linkage map was made using the Map Manager QTX b20 software (Manly et al., 2001), following chicken consensus map 2005 (Schmid et al., 2005) on ArkDB database (http://www.thearkdb. org/) and the report of Takahashi et al. (2005). Total map distance was estimated to be $2,696 \mathrm{cM}$ by using Kosambi map function, covering approximately $68 \%$ of the chicken genome (Schmid et al., 2005).

\section{Statistical Analysis}

Before QTL analyses, phenotypic data were corrected for the effects of two environmental factors, date of hatch and $F_{1}$ dams, using the JMP software version 5.0.1a (SAS Institute Inc.). Phenotypic distributions were also tested whether or not they fit normal distribution by the Shapiro-Wilk's test. Phenotypes of non-normal distribution were approximated to normal distribution using the Box-Cox transformation. In addition, data for the first egg stage were corrected for the 
effect of age at the first eggs to remove the effect of hen ages because age at first egg of $\mathrm{F}_{2}$ hens ranged from $156 \mathrm{~d}$ to $292 \mathrm{~d}$ (Goto et al., 2011). In addition, QTL analyses for LLE, LSE, and SW were accomplished using the data corrected by EW. SW was used for the trait corrections of SS, STN, STB, and STE because this parameter was significantly correlated with the eggshell quality $(P<0.05)$. Pearson's correlation coefficients $(r)$ among all phenotypes in each stage were estimated by using JMP.

\section{QTL Analysis}

QTL analyses were performed with the R/qtl software (Broman et al., 2003). Single-QTL and two-QTL genome scans were performed by the Haley-Knott regression (Haley and Knott, 1992). Genome-wide significant (5\%) and suggestive $(10 \%)$ thresholds were estimated by 1,000 permutations for the autosomes. In the $\mathrm{Z}$ chromosome, the significant thresholds that control the genome-wide false positive rate were estimated as described in the method of Broman et al. (2006). For an epistatic interaction between two loci, 5\% and $10 \%$ genome-wide threshold levels were determined by 1,000 permutations according to the method of Broman and Sen (2009).

The percentage of the phenotypic variance explained by a QTL was computed by R/qtl as described by Broman and Sen (2009). To obtain a total contribution of all QTLs detected for any trait to the phenotypic variance, multiple QTL mapping was performed with the R/qtl software.

\section{Results}

\section{Trait Analysis}

Table 2 shows mean values of 33 external egg traits in the Oh-Shamo and White Leghorn and their $F_{1}$ and $F_{2}$ progenies. The values of egg weight (EW and SW) and egg size (LLE and LSE) of the Oh-Shamo were higher than those of the others in the early stage. In the middle and late stages, the values of the Oh-Shamo and $F_{1}$ were higher than those of the White Leghorn and $F_{2}$, except for the LLE values of White Leghorn in the middle and late stages. The $\mathrm{SS}$ value of $\mathrm{F}_{1}$ was constant at approximately $4 \mathrm{~kg} / \mathrm{cm}^{2}$ in all stages. In contrast, the SS values of the White Leghorn and $\mathrm{F}_{2}$ decreased with age, and that of the Oh-Shamo increased. For eggshell colors (SCL, SCR, and SCY), the eggshells of the OhShamo showed darker color than those of the White Leghorn. The values of $F_{1}$ showed mid-parental values in all stages. The values of eggshell thickness (STN, STB, and STE) of the Oh-Shamo and $F_{1}$ were higher than those of the White Leghorn and $F_{2}$ in all stages, except for the STB and STE values of the White Leghorn in the early stage.

The egg weight and size (EW, LLE, and LSE) of the $F_{2}$ significantly increased with hens' age $\left(F_{2,210}=494.710, F_{2,210}\right.$ $=155.775$, and $F_{2,209}=588.146$, respectively, $P<0.0001$ for all traits, one-way repeated measures ANOVA). The SW also significantly increased with age $\left(F_{2,192}=116.357, P<\right.$ 0.0001). Eggshell thickness (STB and STE) remained unchanged throughout the three stages $\left(F_{2,192}=2.180\right.$ and $F_{2,192}$ $=2.649$, respectively, $P>0.05)$, although STN in the middle stage was significantly higher than the other stages $\left(F_{2,192}=\right.$
$11.858, P<0.0001)$. On the other hand, SS significantly decreased in inverse proportion to increasing age $\left(F_{2,198}=\right.$ 79.047, $P<0.0001)$. Eggshell color (SCL, SCR, and SCY) also became lighter and paler with advancing age $\left(F_{2,218}=\right.$ $87.111, F_{2,218}=102.407$, and $F_{2,218}=102.740$, respectively, $P<0.0001$ for all traits).

Phenotypic correlations of external egg traits in the $F_{2}$ hens are shown in Tables 3-5. Here, traits are confined to six categories, egg weight (EW), eggshell weight (SW), eggshell strength (SS), sizes (LLE and LSE), eggshell thickness (STN, STE, and STB), and colors (SCL, SCR, and SCY) in each stage. Within the same categories, phenotypic correlations between LLE and LSE ranged from 0.60 to 0.73 . Phenotypic correlations varied also among STN, STE, and STB (0.67-0.78). On the other hand, phenotypic correlations of SCL vs SCR, and SCL vs SCY were regularly around -0.90 , whereas those of SCR vs SCY were uniformly around 0.90. Among the different categories, phenotypic correlations of EW vs SW, EW vs size, and SW vs size were $0.70-0.79,0.82-0.96$, and $0.49-0.79$, respectively. Phenotypic correlations of SW vs thickness, SW vs SS, and SS vs thickness were $0.54-0.75,0.39-0.45$, and $0.40-0.76$, respectively. Almost all color traits had no significant phenotypic correlations to EW, SW, SS, size, and thickness.

\section{QTL Analysis}

Main-effect QTL: In the present study, no epistatic QTL was discovered for all external egg traits examined. QTLs with main effects on external egg traits were identified on chromosomes 1, 2, 4, 5, 8, 10,11, 12, 17, and $\mathrm{Z}$ as shown in Table 6. For egg weight (E-EW, M-EW, and L-EW), significant QTLs were detected around MCW0095 (26 cM) on chromosome 8. Between MCW0240 (201 cM) and ABR 0622 (234 cM) on chromosome 4, a suggestive QTL for EEW was identified. Each of these QTLs explained 3.94$11.75 \%$ of the phenotypic variances.

For size (M-LLE, E-LSE, and L-LSE), significant and suggestive QTLs were detected at around MCW0154 $(95 \mathrm{cM})$ on the $\mathrm{Z}$ chromosome. Between ADL0372 (0 cM) and ADL0044 $(44 \mathrm{cM})$ on chromosome 12, significant QTLs for E-LLE and E-LSE were identified. Additionally, a suggestive QTL affecting E-LSE was found between ADL0272 (44 cM) and ADL0106 (88 cM) on chromosome 10. Each of these QTLs explained $3.97-5.76 \%$ of the phenotypic variances.

For eggshell weight (E-SW, M-SW, and L-SW), significant QTLs were detected around ABR0119 $(135 \mathrm{cM})$ on the Z chromosome. Around ABR0530 $(0 \mathrm{cM})$ on chromosome 17, a suggestive QTL for E-SW was identified. Each QTL explained $4.22-7.85 \%$ of the phenotypic variances.

For eggshell strength (E-SS and L-SS), significant QTLs were detected between $M C W 0258(26 \mathrm{cM})$ and $A D L 0273$ (73 $\mathrm{cM})$ and around $A D L 0372(0 \mathrm{cM})$ on chromosomes $\mathrm{Z}$ and 12 , respectively. Each QTL contributed to $4.35-7.96 \%$ of the phenotypic variances.

For eggshell thickness (M-STN, L-STN, M-STB, L-STB, and M-STE), significant and suggestive QTLs were found around MCW0305 $(15 \mathrm{cM})$ and MCW0095 $(26 \mathrm{cM})$ on chromosome 8. Between ABR0119 (135 cM) and ABR0289 (148 
Table 2. Means and standard deviations for 33 external egg traits in the Oh-Shamo and White Leghorn breeds and their $F_{1}$ and $F_{2}$ hens

\begin{tabular}{|c|c|c|c|c|}
\hline \multirow{2}{*}{ Trait $^{1}$} & \multirow{2}{*}{ Group } & \multicolumn{3}{|c|}{ Mean \pm SD (No. of hens) } \\
\hline & & Early stage & Middle stage & Late stage \\
\hline \multirow[t]{4}{*}{ EW (g) } & Oh-Shamo & $49.8 \pm 5.5^{\mathrm{a}}(39)$ & $53.8 \pm 4.2^{\mathrm{a}}(24)$ & $55.0 \pm 4.3^{\mathrm{a}}(14)$ \\
\hline & White Leghorn & $41.3 \pm 2.8^{\mathrm{b}}(23)$ & $47.4 \pm 2.3^{\mathrm{b}}(20)$ & $48.6 \pm 2.2^{\mathrm{b}}(17)$ \\
\hline & $\mathrm{F}_{1}$ & $43.7 \pm 4.0^{\mathrm{b}}(62)$ & $52.6 \pm 2.7^{\mathrm{a}}(56)$ & $54.5 \pm 2.8^{\mathrm{a}}(51)$ \\
\hline & $\mathrm{F}_{2}$ & $42.2 \pm 4.9^{\mathrm{b}}(387)$ & $47.6 \pm 5.1^{\mathrm{b}}(322)$ & $49.4 \pm 5.1^{\mathrm{b}}(225)$ \\
\hline \multirow[t]{4}{*}{ LLE (mm) } & Oh-Shamo & $53.35 \pm 2.30^{\mathrm{a}}(39)$ & $55.05 \pm 1.47^{\mathrm{a}}(24)$ & $55.20 \pm 1.77^{\mathrm{a}, \mathrm{b}}$ \\
\hline & White Leghorn & $51.66 \pm 1.98^{\mathrm{b}}(23)$ & $53.55 \pm 1.38^{\mathrm{a}, \mathrm{b}}(20)$ & $54.49 \pm 1.50^{\mathrm{a}, \mathrm{b}}(17)$ \\
\hline & $\mathrm{F}_{1}$ & $51.54 \pm 1.76^{\mathrm{b}}(62)$ & $54.25 \pm 1.46^{\mathrm{a}}(56)$ & $55.29 \pm 1.34^{\mathrm{a}}(51)$ \\
\hline & $\mathrm{F}_{2}$ & $51.15 \pm 2.47^{\mathrm{b}}(387)$ & $52.44 \pm 2.27^{\mathrm{b}}(322)$ & $53.66 \pm 2.41^{\mathrm{b}}(225)$ \\
\hline \multirow[t]{4}{*}{$\operatorname{LSE}(\mathrm{mm})$} & Oh-Shamo & $40.74 \pm 1.61^{\mathrm{a}}(39)$ & $41.87 \pm 1.34^{\mathrm{a}}(24)$ & $42.02 \pm 1.82^{\mathrm{a}}(14)$ \\
\hline & White Leghorn & $37.85 \pm 0.72^{\mathrm{c}}(23)$ & $40.04 \pm 0.62^{\mathrm{b}}(20)$ & $40.15 \pm 0.65^{\mathrm{b}}(17)$ \\
\hline & $\mathrm{F}_{1}$ & $38.85 \pm 1.28^{\mathrm{b}}(62)$ & $41.54 \pm 0.74^{\mathrm{a}}(56)$ & $42.06 \pm 0.84^{\mathrm{a}}(51)$ \\
\hline & $\mathrm{F}_{2}$ & $38.50 \pm 1.55^{\mathrm{b}, \mathrm{c}}(387)$ & $40.46 \pm 1.64^{\mathrm{b}}(321)$ & $40.82 \pm 1.59^{\mathrm{b}}(225)$ \\
\hline \multirow{4}{*}{ SW (g) } & Oh-Shamo & $4.8 \pm 0.5^{\mathrm{a}}(35)$ & $5.0 \pm 0.6^{\mathrm{a}}(25)$ & $5.2 \pm 0.5^{\mathrm{a}}(15)$ \\
\hline & White Leghorn & $4.2 \pm 0.4^{\mathrm{b}, \mathrm{c}}(7)$ & $4.4 \pm 0.3^{\mathrm{b}}(20)$ & $4.5 \pm 0.3^{\mathrm{b}}(20)$ \\
\hline & $\mathrm{F}_{1}$ & $4.4 \pm 0.3^{\mathrm{b}}(52)$ & $5.2 \pm 0.3^{\mathrm{a}}(58)$ & $5.2 \pm 0.3^{\mathrm{a}}(52)$ \\
\hline & $\mathrm{F}_{2}$ & $4.2 \pm 0.5^{\mathrm{c}}(371)$ & $4.5 \pm 0.5^{\mathrm{b}}(311)$ & $4.6 \pm 0.5^{\mathrm{b}}(209)$ \\
\hline \multirow[t]{4}{*}{$\mathrm{SS}\left(\mathrm{kg} / \mathrm{cm}^{2}\right)$} & Oh-Shamo & $3.61 \pm 0.49^{\mathrm{b}}(35)$ & $3.93 \pm 0.73^{\mathrm{a}, \mathrm{b}}(25)$ & $4.16 \pm 0.46^{\mathrm{a}}(15)$ \\
\hline & White Leghorn & $3.67 \pm 0.42^{\mathrm{a}, \mathrm{b}}(7)$ & $3.53 \pm 0.37^{\mathrm{b}}(20)$ & $3.41 \pm 0.36^{\mathrm{b}}(20)$ \\
\hline & $\mathrm{F}_{1}$ & $4.10 \pm 0.38^{\mathrm{a}}(52)$ & $4.16 \pm 0.50^{\mathrm{a}}(58)$ & $4.04 \pm 0.53^{\mathrm{a}}(52)$ \\
\hline & $\mathrm{F}_{2}$ & $3.97 \pm 0.46^{\mathrm{a}}(371)$ & $3.81 \pm 0.52^{\mathrm{b}}(311)$ & $3.49 \pm 0.60^{\mathrm{b}}(217)$ \\
\hline \multirow[t]{4}{*}{ STN (mm) } & Oh-Shamo & $0.358 \pm 0.021^{\mathrm{a}}(35)$ & $0.368 \pm 0.030^{\mathrm{a}}(25)$ & $0.370 \pm 0.020^{\mathrm{a}}(15)$ \\
\hline & White Leghorn & $0.322 \pm 0.011^{\mathrm{b}}(7)$ & $0.336 \pm 0.018^{\mathrm{b}}(20)$ & $0.331 \pm 0.022^{\mathrm{b}}(20)$ \\
\hline & $\mathrm{F}_{1}$ & $0.355 \pm 0.021^{\mathrm{a}}(52)$ & $0.369 \pm 0.021^{\mathrm{a}}(58)$ & $0.362 \pm 0.022^{\mathrm{a}}(52)$ \\
\hline & $\mathrm{F}_{2}$ & $0.334 \pm 0.026^{\mathrm{b}}(371)$ & $0.343 \pm 0.023^{\mathrm{b}}(311)$ & $0.337 \pm 0.025^{\mathrm{b}}(210)$ \\
\hline \multirow[t]{4}{*}{ STB $(\mathrm{mm})$} & Oh-Shamo & $0.356 \pm 0.023^{\mathrm{a}}(35)$ & $0.361 \pm 0.031^{\mathrm{a}}(25)$ & $0.366 \pm 0.023^{\mathrm{a}}(15)$ \\
\hline & White Leghorn & $0.327 \pm 0.012^{\mathrm{b}, \mathrm{c}}(7)$ & $0.329 \pm 0.019^{\mathrm{b}}(19)$ & $0.327 \pm 0.019^{\mathrm{b}}(20)$ \\
\hline & $\mathrm{F}_{1}$ & $0.344 \pm 0.016^{\mathrm{a}, \mathrm{b}}(52)$ & $0.361 \pm 0.024^{\mathrm{a}}(58)$ & $0.356 \pm 0.024^{\mathrm{a}}(52)$ \\
\hline & $\mathrm{F}_{2}$ & $0.323 \pm 0.024^{\mathrm{c}}(371)$ & $0.326 \pm 0.026^{\mathrm{b}}(311)$ & $0.321 \pm 0.029^{\mathrm{b}}(210)$ \\
\hline \multirow[t]{4}{*}{ STE (mm) } & Oh-Shamo & $0.347 \pm 0.025^{\mathrm{a}}(35)$ & $0.351 \pm 0.029^{\mathrm{b}}(25)$ & $0.351 \pm 0.032^{\mathrm{a}}(15)$ \\
\hline & White Leghorn & $0.332 \pm 0.018^{\mathrm{a}, \mathrm{b}}(7)$ & $0.329 \pm 0.019^{\mathrm{c}}(19)$ & $0.327 \pm 0.020^{\mathrm{b}}(20)$ \\
\hline & $\mathrm{F}_{1}$ & $0.356 \pm 0.020^{\mathrm{a}}(52)$ & $0.374 \pm 0.022^{\mathrm{a}}(58)$ & $0.361 \pm 0.017^{\mathrm{a}}(52)$ \\
\hline & $\mathrm{F}_{2}$ & $0.334 \pm 0.024^{\mathrm{b}}(371)$ & $0.338 \pm 0.024^{\mathrm{c}}(311)$ & $0.334 \pm 0.025^{\mathrm{b}}(210)$ \\
\hline \multirow[t]{4}{*}{$\mathrm{SCL}$} & Oh-Shamo & $75.19 \pm 3.14^{\mathrm{c}}(21)$ & $77.68 \pm 4.91^{\mathrm{d}}(23)$ & $80.14 \pm 4.24^{\mathrm{d}}(14)$ \\
\hline & White Leghorn & $85.98 \pm 2.02^{\mathrm{a}}(12)$ & $88.00 \pm 1.31^{\mathrm{a}}(20)$ & $87.28 \pm 1.39^{\mathrm{a}}(17)$ \\
\hline & $\mathrm{F}_{1}$ & $81.05 \pm 2.86^{\mathrm{b}}(22)$ & $84.04 \pm 2.51^{\mathrm{c}}(56)$ & $83.78 \pm 2.59^{\mathrm{c}}(51)$ \\
\hline & $\mathrm{F}_{2}$ & $83.98 \pm 2.93^{\mathrm{a}}(388)$ & $85.27 \pm 2.93^{\mathrm{b}}(323)$ & $85.09 \pm 2.84^{\mathrm{b}}(236)$ \\
\hline \multirow[t]{4}{*}{$\mathrm{SCR}$} & Oh-Shamo & $6.85 \pm 2.39^{\mathrm{a}}(21)$ & $5.06 \pm 3.07^{\mathrm{a}}(23)$ & $3.93 \pm 2.94^{\mathrm{a}}(14)$ \\
\hline & White Leghorn & $0.20 \pm 1.07^{\mathrm{d}}(12)$ & $-0.30 \pm 0.31^{\mathrm{d}}(20)$ & $-0.29 \pm 0.37^{\mathrm{d}}(17)$ \\
\hline & $\mathrm{F}_{1}$ & $3.80 \pm 1.62^{\mathrm{b}}(22)$ & $2.19 \pm 1.20^{\mathrm{b}}(56)$ & $2.30 \pm 1.26^{\mathrm{b}}(51)$ \\
\hline & $\mathrm{F}_{2}$ & $1.98 \pm 1.52^{\mathrm{c}}(388)$ & $1.34 \pm 1.39^{\mathrm{c}}(322)$ & $1.29 \pm 1.33^{\mathrm{c}}(236)$ \\
\hline \multirow[t]{4}{*}{ SCY } & Oh-Shamo & $22.10 \pm 2.67^{\mathrm{a}}(21)$ & $20.06 \pm 4.04^{\mathrm{a}}(23)$ & $17.54 \pm 3.32^{\mathrm{a}}(14)$ \\
\hline & White Leghorn & $8.27 \pm 3.20^{\mathrm{d}}(12)$ & $5.57 \pm 2.10^{\mathrm{d}}(20)$ & $5.34 \pm 1.86^{\mathrm{d}}(17)$ \\
\hline & $\mathrm{F}_{1}$ & $16.56 \pm 2.25^{\mathrm{b}}(22)$ & $13.86 \pm 2.96^{\mathrm{b}}(56)$ & $14.68 \pm 2.78^{\mathrm{b}}(51)$ \\
\hline & $\mathrm{F}_{2}$ & $12.44 \pm 3.33^{\mathrm{c}}(388)$ & $10.82 \pm 3.73^{\mathrm{c}}(322)$ & $10.89 \pm 3.84^{\mathrm{c}}(236)$ \\
\hline
\end{tabular}

\footnotetext{
${ }^{1}$ Early stage $=$ first 10 eggs, Middle stage $=$ eggs produced during two weeks from 300 days of age, Late stage $=$ eggs produced during two weeks from 400 days of age, EW=egg weight, LLE =length of the long axis of the egg, LSE $=$ length of the short axis of the egg, SW=eggshell weight, $\mathrm{SS}=$ eggshell strength, STN=eggshell thickness at the narrow end of the egg, STB = eggshell thickness at the blunt end of the egg, STE= eggshell thickness at the equator of the egg, SCL $=$ lightness of the eggshell color, $\mathrm{SCR}=$ redness of the eggshell color, SCY=yellowness of the eggshell color.

${ }^{\mathrm{a}-\mathrm{d}}$ Means with the same superscript letter are not significantly different among the groups at $P>0.05$ in each trait within the same stage (one-way ANOVA followed by Tukey’s HSD test).
} 
Table 3. Phenotypic correlations of external egg traits at the early stage in $\mathbf{F}_{\mathbf{2}}$ hens

\begin{tabular}{ccccccccccc}
\hline \hline Trait & E-EW & E-LLE & E-LSE & E-SW & E-SS & E-STN & E-STB & E-STE & E-SCL & E-SCR \\
\hline E-EW & & & & & & & & & & \\
E-LLE & 0.88 & & & & & & & & \\
E-LSE & 0.96 & 0.73 & & & & & & & \\
E-SW & 0.79 & 0.60 & 0.79 & & & & & & \\
E-SS & $0.07^{\text {ns }}$ & $-0.07^{\text {ns }}$ & 0.10 & 0.45 & & & & & \\
E-STN & 0.18 & $-0.02^{\text {ns }}$ & 0.23 & 0.59 & 0.60 & & & & \\
E-STB & 0.20 & $-0.01^{\text {ns }}$ & 0.25 & 0.63 & 0.76 & 0.75 & & & \\
E-STE & 0.26 & 0.05 & 0.23 & 0.75 & 0.62 & 0.78 & 0.78 & & & \\
E-SCL & $0.06^{\text {ns }}$ & $0.09^{\text {ns }}$ & $0.05^{\text {ns }}$ & $-0.09^{\text {ns }}$ & -0.19 & -0.16 & -0.27 & -0.19 & & \\
E-SCR & $-0.07^{\text {ns }}$ & -0.13 & $-0.04^{\text {ns }}$ & $0.11^{\text {ns }}$ & 0.20 & 0.23 & 0.30 & 0.23 & -0.95 & \\
E-SCY & $-0.05^{\text {ns }}$ & $-0.14^{\text {ns }}$ & $-0.01^{\text {ns }}$ & 0.12 & 0.19 & 0.24 & 0.30 & 0.25 & -0.92 & 0.93
\end{tabular}

Trait abbreviations are shown in Materials and Methods.

${ }^{\text {ns }}$ Not significant at $P>0.05$.

Table 4. Phenotypic correlations of external egg traits at the middle stage in $\mathbf{F}_{\mathbf{2}}$ hens

\begin{tabular}{lcccccccccc}
\hline \hline \multicolumn{1}{c}{ Trait } & M-EW & M-LLE & M-LSE & M-SW & M-SS & M-STN & M-STB & M-STE & M-SCL & M-SCR \\
\hline M-EW & & & & & & & & & & \\
M-LLE & 0.82 & & & & & & & & & \\
M-LSE & 0.95 & 0.64 & & & & & & & & \\
M-SW & 0.74 & 0.57 & 0.66 & & & & & & \\
M-SS & $0.12^{\text {ns }}$ & $0.02^{\text {ns }}$ & $0.11^{\text {ns }}$ & 0.42 & & & & & \\
M-STN & $0.05^{\text {ns }}$ & $-0.06^{\text {ns }}$ & $0.03^{\text {ns }}$ & 0.56 & 0.43 & & & & \\
M-STB & $0.11^{\text {ns }}$ & $-0.02^{\text {ns }}$ & $0.09^{\text {ns }}$ & 0.61 & 0.61 & 0.67 & & & \\
M-STE & 0.14 & $0.02^{\text {ns }}$ & $0.10^{\text {ns }}$ & 0.72 & 0.48 & 0.73 & 0.74 & & \\
M-SCL & $0.02^{\text {ns }}$ & $0.05^{\text {ns }}$ & $0.04^{\text {ns }}$ & $-0.07^{\text {ns }}$ & $0.01^{\text {ns }}$ & -0.11 & $-0.10^{\text {ns }}$ & $-0.03^{\text {ns }}$ & \\
M-SCR & -0.09 & -0.11 & -0.10 & $0.01^{\text {ns }}$ & $-0.07^{\text {ns }}$ & 0.14 & 0.08 & 0.04 & -0.92 & \\
M-SCY & $-0.04^{\text {ns }}$ & $-0.07^{\text {ns }}$ & $-0.05^{\text {ns }}$ & $0.02^{\text {ns }}$ & $-0.05^{\text {ns }}$ & 0.11 & $0.07^{\text {ns }}$ & $0.01^{\text {ns }}$ & -0.88 & 0.90 \\
\hline
\end{tabular}

Trait abbreviations are shown in Materials and Methods.

${ }^{n s}$ Not significant at $P>0.05$.

Table 5. Phenotypic correlations of external egg traits at the late stage in $\mathbf{F}_{\mathbf{2}}$ hens

\begin{tabular}{|c|c|c|c|c|c|c|c|c|c|c|c|}
\hline Trait & L-EW & L-LLE & L-LSE & L-SW & L-SS & L-STN & L-STB & L-STE & L-SCL & L-SCR & L-SCY \\
\hline \multicolumn{12}{|l|}{ L-EW } \\
\hline L-LLE & 0.82 & & & & & & & & & & \\
\hline L-LSE & 0.94 & 0.60 & & & & & & & & & \\
\hline L-SW & 0.70 & 0.49 & 0.64 & & & & & & & & \\
\hline L-SS & $0.05^{\mathrm{ns}}$ & $-0.04^{\mathrm{ns}}$ & $0.04^{\mathrm{ns}}$ & 0.39 & & & & & & & \\
\hline L-STN & $0.01^{\mathrm{ns}}$ & $-0.15^{\mathrm{ns}}$ & $0.02^{\mathrm{ns}}$ & 0.54 & 0.41 & & & & & & \\
\hline L-STB & $0.04^{\mathrm{ns}}$ & $-0.14^{\mathrm{ns}}$ & $0.04^{\mathrm{ns}}$ & 0.57 & 0.49 & 0.70 & & & & & \\
\hline L-STE & 0.09 & $-0.05^{\mathrm{ns}}$ & $0.07^{\mathrm{ns}}$ & 0.68 & 0.40 & 0.74 & 0.73 & & & & \\
\hline L-SCL & $0.08^{\mathrm{ns}}$ & $0.06^{\mathrm{ns}}$ & $0.09^{\mathrm{ns}}$ & $0.02^{\mathrm{ns}}$ & $0.04^{\mathrm{ns}}$ & $-0.12^{\mathrm{ns}}$ & $-0.01^{\mathrm{ns}}$ & $-0.05^{\mathrm{ns}}$ & & & \\
\hline L-SCR & $-0.09^{\mathrm{ns}}$ & $-0.09^{\mathrm{ns}}$ & $-0.09^{\mathrm{ns}}$ & $0.03^{\mathrm{ns}}$ & $0.04^{\mathrm{ns}}$ & 0.22 & $0.08^{\mathrm{ns}}$ & $0.12^{\mathrm{ns}}$ & -0.91 & & \\
\hline L-SCY & $-0.03^{\mathrm{ns}}$ & $-0.02^{\mathrm{ns}}$ & $-0.06^{\mathrm{ns}}$ & $0.05^{\mathrm{ns}}$ & $0.05^{\mathrm{ns}}$ & 0.16 & $0.05^{\mathrm{ns}}$ & $0.07^{\mathrm{ns}}$ & -0.89 & 0.90 & \\
\hline
\end{tabular}

Trait abbreviations are shown in Materials and Methods.

${ }^{\text {ns }}$ Not significant at $P>0.05$. 
Table 6. Main-effect QTLs affecting external egg traits

\begin{tabular}{|c|c|c|c|c|c|c|c|c|c|c|c|}
\hline Trait $^{1}$ & Stage $^{2}$ & Chr. & Position $^{3}$ & Flanking markers & $\mathrm{CI}^{4}$ & $\mathrm{LOD}^{5}$ & $\%$ Var. $^{6}$ & Add. $^{7}$ & Dom. ${ }^{8}$ & Differences ${ }^{9}$ & $\begin{array}{l}\text { Total } \\
\text { contribu- } \\
\text { tion }^{10}\end{array}$ \\
\hline \multirow[t]{4}{*}{ EW } & E & 4 & 213 & MCW0240-ABR0622 & $154-250$ & $3.38^{\dagger}$ & 3.94 & 0.25 & 0.11 & $\mathrm{~S}>\mathrm{H}>\mathrm{W}$ & \\
\hline & E & 8 & 40 & MCW0095-ADL0345 & $29-49$ & $6.70^{*}$ & 7.66 & -0.19 & 0.37 & $\mathrm{H} \geq \mathrm{W}>\mathrm{S}$ & 12.18 \\
\hline & M & 8 & 39 & $M C W 0095-A D L 0345$ & $30-47$ & $8.74 *$ & 11.75 & -0.27 & 0.48 & $\mathrm{H} \geq \mathrm{W}>\mathrm{S}$ & 11.75 \\
\hline & $\mathrm{L}$ & 8 & 35 & $M C W 0305-M C W 0095$ & $13-47$ & $5.05^{*}$ & 9.82 & -0.24 & 0.46 & $\mathrm{H} \geq \mathrm{W}>\mathrm{S}$ & 9.82 \\
\hline \multirow[t]{2}{*}{ LLE } & E & 12 & 13 & $A D L 0372-A D L 0044$ & $0-38$ & $4.37^{*}$ & 5.07 & -0.19 & 0.01 & $\mathrm{~W}>\mathrm{H}>\mathrm{S}$ & 5.07 \\
\hline & M & $\mathrm{Z}$ & 100 & MCW0154-LEI0121 & $80-118$ & $3.29 *$ & 4.60 & 0.12 & - & $S>W$ & 4.60 \\
\hline \multirow[t]{4}{*}{ LSE } & $\mathrm{E}$ & 10 & 4 & $A D L 0272-A D L 0106$ & $0-38$ & $3.46^{\dagger}$ & 4.03 & 0.08 & 0.03 & $\mathrm{~S} \geq \mathrm{H}>\mathrm{W}$ & \\
\hline & E & 12 & 24 & $A D L 0372-A D L 0044$ & $0-49$ & $4.99 *$ & 5.76 & 0.14 & 0.03 & $\mathrm{~S}>\mathrm{H}>\mathrm{W}$ & \\
\hline & E & $\mathrm{Z}$ & 85 & ADL0201-MCW0154 & $75-106$ & $3.40 *$ & 3.97 & -0.05 & - & $\mathrm{W}>\mathrm{S}$ & 13.30 \\
\hline & $\mathrm{L}$ & $\mathrm{Z}$ & 104 & MCW0154-LEI0121 & $0-127$ & $2.71^{\dagger}$ & 5.39 & -0.08 & - & $\mathrm{W}>\mathrm{S}$ & 5.39 \\
\hline \multirow[t]{4}{*}{ SW } & E & 17 & 0 & ABR0530 & $0-23$ & $3.37^{\dagger}$ & 4.22 & -0.16 & 0.06 & $\mathrm{~W} \geq \mathrm{H}>\mathrm{S}$ & \\
\hline & $\mathrm{E}$ & $\mathrm{Z}$ & 144 & ABR0119-ABR0289 & $118-148$ & $6.39 *$ & 7.85 & 0.16 & - & $S>W$ & 10.60 \\
\hline & M & Z & 130 & LEI0121-ABR0119 & $112-148$ & $5.46^{*}$ & 7.77 & 0.18 & - & $\mathrm{S}>\mathrm{W}$ & 7.77 \\
\hline & $\mathrm{L}$ & Z & 148 & ABR0119-ABR0289 & $113-148$ & $3.62 *$ & 7.85 & 0.18 & - & $S>W$ & 7.85 \\
\hline \multirow[t]{2}{*}{ SS } & $\mathrm{E}$ & $\mathrm{Z}$ & 51 & $M C W 0258-A D L 0273$ & $34-77$ & $3.49 *$ & 4.35 & 0.17 & - & $S>W$ & 4.35 \\
\hline & $\mathrm{L}$ & 12 & 0 & $A D L 0372$ & $0-21$ & $3.77 *$ & 7.96 & -0.27 & -0.32 & $\mathrm{~W}>\mathrm{S} \geq \mathrm{H}$ & 7.96 \\
\hline \multirow[t]{5}{*}{ STN } & E & 5 & 3 & MCW0038-MCW0214 & $0-21$ & $3.29^{\dagger}$ & 4.00 & 0.15 & 0.23 & $\mathrm{H} \geq \mathrm{S}>\mathrm{W}$ & \\
\hline & $\mathrm{E}$ & 17 & 18 & ADL0293-MCW0330 & $6-34$ & $3.74 *$ & 4.53 & -0.15 & -0.23 & $\mathrm{~W}>\mathrm{S} \geq \mathrm{H}$ & \\
\hline & $\mathrm{E}$ & $\mathrm{Z}$ & 146 & ABR0119-ABR0289 & $36-148$ & $3.28 *$ & 3.99 & 0.15 & - & $\mathrm{S}>\mathrm{W}$ & 10.50 \\
\hline & M & 8 & 41 & $M C W 0095-A D L 0345$ & $31-70$ & $3.52^{\dagger}$ & 5.08 & 0.13 & -0.30 & $\mathrm{~S}>\mathrm{W} \geq \mathrm{H}$ & 5.08 \\
\hline & $\mathrm{L}$ & 8 & 44 & MCW0095-ADL0345 & $32-55$ & $4.20 *$ & 8.85 & 0.08 & -0.56 & $\mathrm{~S}>\mathrm{W}>\mathrm{H}$ & 8.85 \\
\hline \multirow[t]{4}{*}{ STB } & $\mathrm{E}$ & Z & 144 & ABR0119-ABR0289 & $115-148$ & $3.09 *$ & 3.09 & 0.17 & - & $\mathrm{S}>\mathrm{W}$ & 3.09 \\
\hline & M & 8 & 35 & MCW0305-MCW0095 & $26-52$ & $6.13^{*}$ & 8.68 & 0.27 & -0.23 & $\mathrm{~S}>\mathrm{H} \geq \mathrm{W}$ & \\
\hline & M & Z & 141 & ABR0119-ABR0289 & $118-148$ & $3.23 *$ & 4.67 & 0.15 & - & $\mathrm{S}>\mathrm{W}$ & 13.10 \\
\hline & $\mathrm{L}$ & 8 & 15 & ABR0017-MCW0305 & $0-55$ & $3.50^{\dagger}$ & 7.41 & 0.21 & -0.41 & $\mathrm{~S}>\mathrm{W} \geq \mathrm{H}$ & 7.41 \\
\hline \multirow[t]{3}{*}{ STE } & $\mathrm{E}$ & 17 & 8 & $A B R 0530-A D L 0293$ & $0-30$ & $3.90^{*}$ & 4.85 & -0.21 & -0.08 & $\mathrm{~W}>\mathrm{H} \geq \mathrm{S}$ & \\
\hline & E & $\mathrm{Z}$ & 141 & ABR0119-ABR0289 & $118-148$ & $5.90 *$ & 7.25 & 0.17 & - & $\mathrm{S}>\mathrm{W}$ & 10.90 \\
\hline & M & 8 & 20 & ABR0017-MCW0305 & $2-44$ & $3.81^{*}$ & 5.49 & 0.25 & -0.04 & $\mathrm{~S}>\mathrm{H}>\mathrm{W}$ & 5.49 \\
\hline \multirow[t]{2}{*}{ SCL } & M & 5 & 71 & $M C W 0223-A D L 0233$ & $57-83$ & $4.59 *$ & 6.33 & -0.33 & -0.09 & $\mathrm{~W}>\mathrm{H}>\mathrm{S}$ & 6.33 \\
\hline & $\mathrm{L}$ & 5 & 73 & $M C W 0223-A D L 0233$ & $62-84$ & $5.86^{*}$ & 10.81 & -0.42 & 0.02 & $\mathrm{~W}>\mathrm{H}>\mathrm{S}$ & 10.81 \\
\hline \multirow[t]{6}{*}{ SCR } & E & 1 & 138 & MCW0112-MCW0058 & $114-155$ & $4.31 *$ & 4.99 & 0.29 & 0.07 & $\mathrm{~S}>\mathrm{H}>\mathrm{W}$ & \\
\hline & $\mathrm{E}$ & 4 & 186 & ABR0331-MCW0240 & $146-255$ & $3.80^{*}$ & 4.42 & 0.24 & 0.23 & $\mathrm{~S} \geq \mathrm{H}>\mathrm{W}$ & \\
\hline & $\mathrm{E}$ & 5 & 69 & $M C W 0223-A D L 0233$ & $45-85$ & $3.61^{\dagger}$ & 4.20 & 0.26 & 0.14 & $\mathrm{~S} \geq \mathrm{H}>\mathrm{W}$ & 13.36 \\
\hline & M & 1 & 146 & MCW0112-MCW0058 & $123-200$ & $4.12 *$ & 5.73 & 0.32 & -0.12 & $\mathrm{~S}>\mathrm{H}>\mathrm{W}$ & \\
\hline & M & 5 & 71 & $M C W 0223-A D L 0233$ & $52-86$ & $4.19^{*}$ & 5.81 & 0.32 & 0.10 & $\mathrm{~S}>\mathrm{H}>\mathrm{W}$ & 11.69 \\
\hline & $\mathrm{L}$ & 5 & 69 & $M C W 0223-A D L 0233$ & $50-80$ & $6.07^{*}$ & 11.17 & 0.44 & -0.17 & $\mathrm{~S}>\mathrm{H}>\mathrm{W}$ & 11.17 \\
\hline \multirow[t]{8}{*}{ SCY } & $\mathrm{E}$ & 1 & 137 & MCW0112-MCW0058 & $119-223$ & $3.95^{*}$ & 4.58 & 0.26 & 0.11 & $\mathrm{~S} \geq \mathrm{H}>\mathrm{W}$ & \\
\hline & E & 4 & 183 & ABR0331-MCW0240 & $147-261$ & $3.82 *$ & 4.43 & 0.22 & 0.23 & $\mathrm{~S} \geq \mathrm{H}>\mathrm{W}$ & \\
\hline & $\mathrm{E}$ & 5 & 73 & $M C W 0223-A D L 0233$ & $60-84$ & $5.72 *$ & 6.56 & 0.31 & 0.11 & $\mathrm{~S}>\mathrm{H}>\mathrm{W}$ & 15.28 \\
\hline & M & 1 & 150 & $M C W 0112-M C W 0058$ & $133-205$ & $4.27^{*}$ & 5.92 & 0.32 & -0.06 & $\mathrm{~S}>\mathrm{H}>\mathrm{W}$ & \\
\hline & M & 2 & 0 & LEI0124 & $0-52$ & $3.34^{\dagger}$ & 4.67 & 0.24 & 0.17 & $\mathrm{~S} \geq \mathrm{H}>\mathrm{W}$ & \\
\hline & M & 5 & 72 & $M C W 0223-A D L 0233$ & $60-84$ & $5.29 *$ & 7.29 & 0.35 & 0.10 & $\mathrm{~S}>\mathrm{H}>\mathrm{W}$ & \\
\hline & M & 11 & 34 & ADL0210-MCW0066 & $20-49$ & $3.97 *$ & 5.53 & -0.27 & -0.15 & $\mathrm{~W}>\mathrm{H} \geq \mathrm{S}$ & 21.34 \\
\hline & $\mathrm{L}$ & 5 & 73 & $M C W 0223-A D L 0233$ & $64-83$ & $8.56^{*}$ & 15.39 & 0.52 & -0.09 & $\mathrm{~S}>\mathrm{H}>\mathrm{W}$ & 15.39 \\
\hline
\end{tabular}

${ }^{1} \mathrm{EW}=$ egg weight, $\mathrm{LLE}=$ length of the long axis of the egg, $\mathrm{LSE}=$ length of the short axis of the egg, $\mathrm{SW}=$ eggshell weight, $\mathrm{STN}=$ eggshell thickness at the narrow end of the egg, STE=eggshell thickness at the equator of the egg, STB=eggshell thickness at the blunt end of the egg, SCL $=$ lightness of the eggshell color, $\mathrm{SCR}=$ redness of the eggshell color, SCY=yellowness of the eggshell color.

${ }^{2} \mathrm{E}-=$ early stage (first 10 eggs), $\mathrm{M}-=$ middle stage (during two weeks from 300 days of age), $\mathrm{L}-=$ late stage (during two weeks from 400 days of age).

${ }^{3}$ Chromosomal position (cM) in the present study.

${ }^{4} 1.5$-LOD drop confidence interval in cM.

${ }^{5}$ Genome-wide significant and suggestive QTLs detected $\left(*=5 \%\right.$ level, ${ }^{\dagger}=10 \%$ level).

${ }^{6}$ Percentage of the phenotypic variance explained by the QTL.

${ }^{7}$ Additive effect of the QTL shown in standard deviation unit. Positive and negative signs show that the Oh-Shamo (S) allele increased and decreased phenotypic values, respectively.

${ }^{8}$ Dominance effect of the QTL shown in standard deviation unit.

${ }^{9}$ Phenotypic differences among three possible genotypes at the nearest marker locus, two homozygotes for either the Oh-Shamo (S) or White Leghorn (W) allele and heterozygote (H), estimated by one-way ANOVA (followed by Tukey's HSD test).

${ }^{10}$ A total contribution of all QTLs detected for the trait to the phenotypic variance, shown in \%. 
Table 7. Summary of the positions, target traits, and expression stages of the QTLs detected in the present study

\begin{tabular}{|c|c|c|c|c|c|c|c|c|c|c|}
\hline \multirow[t]{2}{*}{ QTL no. } & \multirow[t]{2}{*}{ Chr. } & \multirow[t]{2}{*}{ Markers (position, $\mathrm{cM})^{1}$} & \multirow[t]{2}{*}{ Trait categories $^{2}$} & \multirow[t]{2}{*}{ Traits $^{2}$} & \multicolumn{3}{|c|}{$\begin{array}{l}\text { Stage of QTL } \\
\text { expression }^{3}\end{array}$} & \multicolumn{3}{|c|}{$\begin{array}{c}\text { Phenotypic correlations } \\
\text { between stages }\end{array}$} \\
\hline & & & & & $\mathrm{E}$ & M & $\mathrm{L}$ & E-M & E-L & M-L \\
\hline \multirow[t]{2}{*}{ QTL1 } & 1 & $M C W 0112(205)-M C W 0058(241)$ & eggshell color & SCR & $\mathrm{X}$ & $\mathrm{X}$ & & 0.85 & 0.77 & 0.87 \\
\hline & & & & SCY & $\mathrm{X}$ & $\mathrm{X}$ & & 0.85 & 0.79 & 0.86 \\
\hline QTL2 & 2 & Around LEIO124 (0*) & eggshell color & SCY & & $\mathrm{X}^{\dagger}$ & & 0.85 & 0.79 & 0.86 \\
\hline \multirow[t]{3}{*}{ QTL3 } & 4 & Around $M C W 0240(201)$ & egg weight & EW & $\mathrm{X}^{\dagger}$ & & & 0.74 & 0.62 & 0.88 \\
\hline & & & eggshell color & SCR & $\mathrm{X}$ & & & 0.85 & 0.77 & 0.87 \\
\hline & & & & SCY & $\mathrm{X}$ & & & 0.85 & 0.79 & 0.86 \\
\hline QTL4 & 5 & $M C W 0038(71)-M C W 0214(88)$ & eggshell thickness & STN & $\mathrm{X}^{\dagger}$ & & & 0.68 & 0.43 & 0.61 \\
\hline \multirow[t]{3}{*}{ QTL5 } & 5 & $M C W 0223(123)-A D L 0233(151)$ & eggshell color & SCL & & $\mathrm{X}$ & $\mathrm{X}$ & 0.82 & 0.78 & 0.86 \\
\hline & & & & SCR & $\mathrm{X}$ & $\mathrm{X}$ & $\mathrm{X}$ & 0.85 & 0.77 & 0.87 \\
\hline & & & & SCY & $\mathrm{X}$ & $\mathrm{X}$ & $\mathrm{X}$ & 0.85 & 0.79 & 0.86 \\
\hline \multirow[t]{4}{*}{ QTL6 } & 8 & Around $M C W 0305$ (15) and $M C W 0095$ (26) & egg weight & EW & $\mathrm{X}$ & $\mathrm{X}$ & $\mathrm{X}$ & 0.74 & 0.62 & 0.88 \\
\hline & & & eggshell thickness & STN & & $\mathrm{X}$ & $\mathrm{X}$ & 0.68 & 0.43 & 0.61 \\
\hline & & & & STB & & $\mathrm{X}$ & $\mathrm{X}$ & 0.57 & 0.35 & 0.63 \\
\hline & & & & STE & & $X$ & & 0.62 & 0.52 & 0.71 \\
\hline QTL7 & 10 & $A D L 0272(44)-A D L 0106(88)$ & egg size & LSE & $\mathrm{X}^{\dagger}$ & & & 0.76 & 0.66 & 0.87 \\
\hline QTL8 & 11 & $A D L 0210(54)-M C W 0066$ (69) & eggshell color & SCY & & $\mathrm{X}$ & & 0.85 & 0.79 & 0.86 \\
\hline \multirow[t]{3}{*}{ QTL9 } & 12 & Around $A D L 0372(0)$ & egg size & LLE & $X$ & & & 0.61 & 0.50 & 0.82 \\
\hline & & & & LSE & $\mathrm{X}$ & & & 0.76 & 0.66 & 0.87 \\
\hline & & & eggshell strength & SS & & & $\mathrm{X}$ & 0.41 & 0.31 & 0.55 \\
\hline \multirow[t]{3}{*}{ QTL10 } & 17 & Around ADL0293 (26) & eggshell weight & SW & $\mathrm{X}^{\dagger}$ & & & 0.70 & 0.54 & 0.77 \\
\hline & & & eggshell thickness & STN & $\mathrm{X}$ & & & 0.68 & 0.43 & 0.61 \\
\hline & & & & STE & $\mathrm{X}$ & & & 0.62 & 0.52 & 0.71 \\
\hline QTL11 & $\mathrm{Z}$ & Around $A D L 0273$ (73) & eggshell strength & SS & $\mathrm{X}$ & & & 0.41 & 0.31 & 0.55 \\
\hline \multirow[t]{2}{*}{ QTL12 } & $\mathrm{Z}$ & Around $M C W 0154$ (95) & egg size & LLE & & $\mathrm{X}$ & & 0.61 & 0.50 & 0.82 \\
\hline & & & & LSE & $\mathrm{X}$ & & $\mathrm{X}$ & 0.76 & 0.66 & 0.87 \\
\hline \multirow[t]{4}{*}{ QTL13 } & $\mathrm{Z}$ & Around $A B R 0119\left(135^{*}\right)$ & eggshell weight & SW & $\mathrm{X}$ & $\mathrm{X}$ & $\mathrm{X}$ & 0.70 & 0.54 & 0.77 \\
\hline & & & eggshell thickness & STN & $\mathrm{X}$ & & & 0.68 & 0.43 & 0.61 \\
\hline & & & & STB & $X$ & $\mathrm{X}$ & & 0.57 & 0.35 & 0.63 \\
\hline & & & & STE & $\mathrm{X}$ & & & 0.62 & 0.52 & 0.71 \\
\hline
\end{tabular}

\footnotetext{
${ }^{1}$ Markers close to QTL and their reference positions. * indicates approximate position because the marker has no reference map position.

${ }^{2}$ Traits were classified into six categories, i.e. egg weight (EW), egg size (LLE and LSE), eggshell weight (SW), eggshell thickness (STN, STB, and STE), eggshell strength (SS), and eggshell color (SCL, SCR, and SCY). Trait abbreviations are shown in Materials and Methods.

${ }^{3} \mathrm{X}$ indicates the stage when the QTL was detected. $\mathrm{E}=$ early stage (first 10 eggs), $\mathrm{M}=$ middle stage (during two weeks from 300 days of age), $\mathrm{L}$

$=$ late stage (during two weeks from 400 days of age). ${ }^{\dagger}$ indicates the QTL at suggestive level.
}

$\mathrm{cM}$ ) on the $\mathrm{Z}$ chromosome, significant QTLs influencing ESTN, E-STB, M-STB, and E-STE were discovered. Significant QTLs affecting E-STN and E-STE were identified at around $A D L 0293(26 \mathrm{cM})$ on chromosome 17. In addition, a suggestive QTL for E-STN was found between MCW0038 $(71 \mathrm{cM})$ and $M C W 0214(88 \mathrm{cM})$ on chromosome 5. Each QTL accounted for 3.09-8.85\% of the phenotypic variances.

For eggshell color (M-SCL, L-SCL, E-SCR, M-SCR, LSCR, E-SCY, M-SCY, and L-SCY), significant and suggestive QTLs were detected between MCW0223 $(123 \mathrm{cM})$ and ADL0233 $(151 \mathrm{cM})$ on chromosome 5. Significant QTLs for E-SCR, M-SCR, E-SCY, and M-SCY were discovered between MCW0112 $(205 \mathrm{cM})$ and MCW0058 $(241 \mathrm{cM})$ on chromosome 1. Significant QTLs influencing E-SCR and ESCY were detected between $A B R 0331(183 \mathrm{cM})$ and $M C W$ $0240(201 \mathrm{cM})$ on chromosome 4. Moreover, significant and suggestive QTLs for M-SCY were found between ADL0210 $(54 \mathrm{cM})$ and $M C W 0066(69 \mathrm{cM})$ and around LEIO124 $(0 \mathrm{cM})$ on chromosomes 11 and 2, respectively. Each QTL contributed to $4.20-15.39 \%$ of the phenotypic variances.

Multiple QTL mapping estimated a total contribution of all QTLs detected for each of external egg traits to the phenotypic variances. This contribution varied from 3.09 to $21.34 \%$ depending on the traits.

The QTL alleles derived from the Oh-Shamo uniquely increased most external egg trait values (Table 6), as expected from the higher phenotypic values in the Oh-Shamo than in the White Leghorn (Table 2). However, there were some QTL alleles with opposite effects to phenotypic differences between the Oh-Shamo and the White Leghorn, the phenomenon being called transgressive segregation (Rieseberg et al., 1999). For example, at the EW QTL on chromosome 8, the allele derived from the Oh-Shamo decreased the trait value, in spite of the superior phenotypic value of the OhShamo over the White Leghorn. At the SS QTL on the Z chromosome, the Oh-Shamo allele increased the trait value at 
the early stage, regardless of its inferior phenotypic value to the White Leghorn.

As summarized in Table 7, we found 13 independent QTL regions. They were one QTL each on chromosome 1 (between $M C W 0112$ and MCW0058), on chromosome 2 (around LEI0124), on chromosome 4 (around MCW0240), two QTLs on chromosome 5 (between MCW0038 and MCW0214 and between MCW0223 and ADL0233), one QTL each on chromosome 8 (around MCW0305 and MCW0095), on chromosome 10 (between $A D L 0272$ and $A D L 0106$ ), on chromosome 11 (between $A D L 0210$ and MCW0066), on chromosome 12 (around ADL0372), on chromosome 17 (around ADL0293), and three QTLs on the $\mathrm{Z}$ chromosome (around ADL0273, $M C W 0154$, and ABR0119).

Age-related changes of main-effect QTLs: As shown in Table 7, the present study revealed 13 QTL regions affecting six categories of external egg-related traits, i.e. eggshell color, egg weight, egg size, eggshell thickness, eggshell strength, and eggshell weight. In each trait for which the responsible QTL was detected, moderate to high phenotypic correlations were observed among stages. Irrespective of the correlations, however, the responsible QTL was not always found through all the three stages.

For eggshell colors, QTL5 (see Table 7) affected SCR and SCY at all stages from the early to late, while QTL1 for SCR and SCY was found at early and middle stages and QTL5 for SCL was at middle and late stages. QTL3 affected SCR and SCY at the early stage only, and QTL2 for SCY and QTL8 for SCY only at the middle stage.

For egg weight, QTL6 was detected at all stages from the early to late, while QTL3 was at the early stage only. For egg size, QTL12 for LSE was found at the early and late stages, and the same QTL for LLE was at the middle stage only. QTL7 for LSE and QTL9 for LLE and LSE were at the early stage only.

For eggshell thickness, QTL13 for STB was discovered at early and middle stages, QTL6 for STN and STB at middle and late stages, QTL4 for STN, QTL10 for STN and STE, and QTL13 for STN and STE were found at the early stage only, and QTL6 for STE was at the middle stage only. For eggshell strength, QTL11 was detected for SS at the early stage only, and QTL9 was at the late stage only. For eggshell weight, QTL13 was exhibited at all stages from the early to late. QTL10 did so at the early stage only.

\section{Discussion}

We discovered 13 external egg quality QTLs on chromosomes $1,2,4,5,8,10,11,12,17$, and $\mathrm{Z}$ in the present study. Among them, six QTLs overlapped in their chromosomal positions with the QTLs reported by other research groups. Around QTL3 (see Table 7) on chromosome 4, many researchers have found a QTL that affects egg weight and/or eggshell colors (Tuiskula-Haavisto et al., 2002; Kerje et al., 2003; Sasaki et al., 2004; Schreiweis et al., 2006; Liu et al., 2011). Around QTL6 on chromosome 8, Yao et al. (2010) and Liu et al. (2011) have reported QTLs for egg weight and eggshell thickness. The position of QTL8 on chromosome 11 for eggshell color was similar to that of the QTL found by Sasaki et al. (2004). Around QTL9 on chromosome 12, which affects eggshell strength, TuiskulaHaavisto et al. (2010) have detected QTLs affecting eggshell weight and eggshell strength. QTL11 and QTL13 on the Z chromosome for weight and strength of the eggshell also coincided with previously reported QTLs (TuiskulaHaavisto et al., 2002, 2010; Sasaki et al., 2004). Although there were some overlapping results as described above, the present study found seven novel QTLs. Thus, the accumulation of QTL information with several mapping resources will be very important for understanding the genetic basis of external egg traits.

In the present study, the highest LOD peak was detected for QTL6 on chromosome 8 affecting egg weight (LOD= 8.74). QTL6 also affected eggshell thickness. In addition to our result, several QTLs in this chromosomal region have been reported for egg number, egg shape, and eggshell colors (Tuiskula-Haavisto et al., 2002; Hansen et al., 2005; Yao et al., 2010). Therefore, the QTL region on chromosome 8 should be marked as an important region for the regulation of phenotypic variation of egg traits.

Until now, several QTLs have been reported around the $200 \mathrm{cM}$ region on chromosome 4 (reviewed by Abasht et al., 2006; Hu et al., 2007, 2012). We found QTL3 that affects external egg quality traits (egg weight and eggshell colors) at the region on chromosome 4 , in spite of no significant phenotypic correlations among those traits. Moreover, at the similar region, QTLs for several kinds of egg quality traits (weight, shape, and strength of the eggshell and weight of albumen and yolk) have been reported by Sasaki et al. (2004), Schreiweis et al. (2006), Abasht et al. (2009), and Dunn et al. (2009). In addition to these, at the region on chromosome 4, Tsudzuki et al. (2007) have reported a QTL affecting body weight and shank length. Furthermore, many research groups have also mapped QTLs that affect growth and bone traits around the region (Carlborg et al., 2004; Zhou et al., 2006, 2007; Nadaf et al., 2009; Gu et al., 2011). Therefore, the QTL region may have an important role in bone and egg formation through the calcium metabolic pathway. However, a large number of loci for egg and bone formation would still remain unmapped. Further studies will be required to reveal the genetic control of the traits involved in calcium metabolism.

There were phenotypic correlations among egg weights, egg sizes, eggshell weights, eggshell strengths, and eggshell thicknesses in the present study. Such correlations are likely to occur due to the closely linked loci or pleiotropic loci. We therefore expected prior to QTL mapping that our work would be able to reveal some overlapped loci for these multiple traits. As expected, common QTL regions affecting multiple traits, such as egg weights, egg sizes, eggshell strengths, and eggshell thicknesses were discovered on chromosomes $4,8,12,17$, and $\mathrm{Z}$. These loci may explain a part of the reason why the phenotypic correlations were observed. On the other hand, there were few phenotypic correlations between eggshell colors (lightness, redness, and 
yellowness) and the other external egg traits, similar to the report of Zhang et al. (2005). Thus, prior to QTL mapping, we supposed that the loci affecting eggshell colors would not be consistent with those for the other traits. The present QTL mapping study revealed QTLs on chromosomes 1, 2, 5, and 11 that affect eggshell color traits only, which provided partial evidence that eggshell colors and other external egg traits are controlled by distinct genetic modules.

Among the 13 QTLs detected in the present study, some QTLs expressed in all the three stages and the others did in one or two stage(s) only, irrespective of the existence of positive phenotypic correlations among all the three stages in each trait (Table 7). For example, three QTLs (QTL1, QTL3, and QTL5) were found for the eggshell color trait SCR and high stage correlations $(r=0.77-0.87)$ were observed. As expected, QTL5 exerted its effect on all stages. However, QTL1 expressed in both early and middle stages and QTL3 did in the early stage only. Although the reason why such differences in QTL expression occurred is unclear from the present study, there is a possibility that the differences in contributions/effects of each QTL led to the discrepancy of the QTL discovery.

We here revealed that egg weight increases and eggshell color becomes lighter and paler with increasing hens' age, consistent with previous studies (Roland, 1979; Ledur et al., 2002; Odabasi et al., 2007). In the present study, it was found that the size and strength of eggs in $\mathrm{F}_{2}$ hens increased and decreased, respectively, with aging. Roland (1979) and Odabasi et al. (2007) supposed that the increase of surface area of the eggshell and the decrease of eggshell thickness are closely related to the increase of egg weight, and consequently the eggs at the late stage would have lower eggshell pigmentation and lower eggshell quality. We observed a similar trend from the results of our phenotypic analyses for the White Leghorn. On the other hand, the eggshell strength in the Oh-Shamo gradually increased with aging unexpectedly, in spite of increasing egg size. This suggests that the Oh-Shamo has some unique alleles at QTLs affecting eggshell quality in the late stage. Although the present study revealed two QTLs affecting eggshell strength, these were not enough to explain the phenomena. However, it is thought that the Oh-Shamo, an untapped chicken breed, might have a large potential to improve eggshell quality at the late stage of egg laying.

QTLs for eggshell colors were found on chromosomes 1, 2, 4, 5, and 11. Among them, QTLs on chromosomes 1, 2, 4, and 11 (QTL1, QTL2, QTL3, and QTL8) were expressed at the early and/or middle stage(s). These loci contributed to $4.42-5.92 \%$ of the phenotypic variances. On the other hand, the QTL on chromosome 5 (QTL5) affected lightness at the middle and late stages, and through the early to late stages for redness and yellowness. In other words, the QTL that appeared at the late stage is the chromosome 5 QTL only. Furthermore, it showed relatively large contributions (10.81$15.39 \%$ ) at the late stage. These results suggest that the contributions of the chromosome 5 QTL may be higher, due to progressively smaller effects on the traits at the late stage in QTLs on chromosomes 1, 2, 4, and 11. Consequently, the changes in the contributions/effects of these QTLs may lead to eggshell color becoming lighter and paler with advancing hens' age. The present results suggested that the quality of external egg traits is regulated by multiple loci that shift their contributions depending on age. Hence, QTL analyses with data focused on several production stages will have a large potential to provide further insights of phenotypic changes caused by aging.

We found no epistatic QTLs for external egg quality in the present study. However, some epistatic QTLs have been reported in chickens (Carlborg et al., 2003, 2004, 2006; Cheng et al., 2007; Wahlberg et al., 2009; Ankra-Badu et al., 2010; Tuiskula-Haavisto et al., 2010, Goto et al., 2011). These epistatic loci have substantial effect on phenotypic variance (Carlborg and Haley, 2004). Therefore, QTL mapping studies should be performed with the interaction effects between loci in mind.

In conclusion, 13 QTLs for external egg traits were discovered using the Oh-Shamo, a unique indigenous Japanese breed of chickens. The age-related changes of QTLs illustrate one aspect of the complex genetic basis of external egg traits.

\section{Acknowledgments}

This work was supported in part by a grant in aid for Scientific Research (B) (\#19380159) from the Japan Society for the Promotion of Science to M.T. and A.I. We thank Dr. L.M. Liao for proofreading the manuscript.

\section{References}

Abasht B, Dekkers JCM and Lamont SJ. Review of quantitative trait loci identified in the chicken. Poultry Science, 85: 2079-2096. 2006.

Abasht B, Sandford E, Arango J, Settar P, Fulton JE, O'Sullivan NP, Hassen A, Habier D, Fernando RL, Dekkers JCM and Lamont SJ. Extent and consistency of linkage disequilibrium and identification of DNA markers for production and egg quality traits in commercial layer chicken populations. BMC Genomics, 10 (Suppl 2): S2. 2009.

Ankra-Badu GA, Shriner D, Le Bihan-Duval E, Mignon-Grasteau S, Pitel F, Beaumont C, Duclos MJ, Simon J, Porter TE, Vignal A, Cogburn LA, Allison DB, Yi N and Aggrey SE. Mapping main, epistatic and sex-specific QTL for body composition in a chicken population divergently selected for low or high growth rate. BMC Genomics, 11: 107. 2010.

Broman KW and Sen S. A guide to QTL mapping with R/qtl. Springer, New York. 2009.

Broman KW, Sen S, Owens SE, Manichaikul A, Southard-Smith EM and Churchill GA. The $\mathrm{X}$ chromosome in quantitative trait locus mapping. Genetics, 174: 2151-2158. 2006.

Broman KW, Wu H, Sen S and Churchill GA. R/qtl: QTL mapping in experimental crosses. Bioinformatics, 19: 889-890. 2003.

Carlborg Ö and Haley CS. Epistasis: too often neglected in complex trait studies? Nature Reviews Genetics, 5: 618-625. 2004.

Carlborg Ö, Hocking PM, Burt DW and Haley CS. Simultaneous mapping of epistatic QTL in chickens reveals clusters of QTL pairs with similar genetic effects on growth. Genetical Research, 83: 197-209. 2004 
Carlborg Ö, Jacobsson L, Ahgren P, Siegel P and Andersson L. Epistasis and the release of genetic variation during long-term selection. Nature Genetics, 38: 418-420. 2006.

Carlborg Ö, Kerje S, Schütz K, Jacobsson L, Jensen $\mathrm{P}$ and Andersson L. A global search reveals epistatic interaction between QTL for early growth in the chicken. Genome Research, 13: 413-421. 2003.

Cheng $\mathrm{HH}$, Zhang $\mathrm{Y}$ and Muir WM. Evidence for widespread epistatic interactions influencing Marek's disease virus viremia levels in chicken. Cytogenetic and Genome Research, 117: 313-318. 2007.

Dunn IC, Joseph NT, Bain M, Edmond A, Wilson PW, Milona P, Nys Y, Gautron J, Schmutz M, Preisinger R and Waddington D. Polymorphisms in eggshell organic matrix genes are associated with eggshell quality measurements in pedigree Rhode Island Red hens. Animal Genetics, 40: 110-114. 2009.

Fulton JE. Selection for avian immune response: a commercial breeding company challenge. Poultry Science, 83: 658-661. 2004.

Goto T, Ishikawa A, Onitsuka S, Goto N, Fujikawa Y, Umino T, Nishibori $\mathrm{M}$ and Tsudzuki $\mathrm{M}$. Mapping quantitative trait loci for egg production traits in an $\mathrm{F}_{2}$ intercross of Oh-Shamo and White Leghorn chickens. Animal Genetics, 42: 634-641. 2011.

Gu X, Feng C, Ma L, Song C, Wang Y, Da Y, Li H, Chen K, Ye S, Ge C, Hu X and Li N. Genome-wide association study of body weight in chicken F2 resource population. PLoS One, 6: e21872. 2011.

Haley CS and Knott SA. A simple regression method for mapping quantitative trait loci in line crosses using flanking markers. Heredity, 69: 315-324. 1992.

Hansen C, Yi N, Zhang YM, Xu S, Gavora J and Cheng HH. Identification of QTL for production traits in chickens. Animal Biotechnology, 16: 67-79. 2005.

Hincke MT, Nys Y and Gautron J. The role of matrix proteins in eggshell formation. Journal of Poultry Science, 47: 208-219. 2010 .

Hu Z-L, Fritz ER and Reecy JM. AnimalQTLdb: a livestock QTL database tool set for positional QTL information mining and beyond. Nucleic Acids Research, 35: D604-609. 2007.

Hu Z-L, Park CA, Wu X-L and Reecy JM. Animal QTLdb: an improved database tool for livestock animal QTL/association data dissemination in the post-genome era. Nucleic Acids Research, 41: D871-879. 2013.

Kerje S, Carlborg Ö, Jacobsson L, Schütz K, Hartmann C, Jensen P and Andersson L. The twofold difference in adult size between the red junglefowl and White Leghorn chickens is largely explained by a limited number of QTLs. Animal Genetics, 34: 264-274. 2003.

Ledur MC, Liljedahl LE, McMillan I, Asselstine L and Fairfull RW. Genetic effects of aging on egg quality traits in the first laying cycle of White Leghorn strains and strain crosses. Poultry Science, 81: 1439-1447. 2002.

Liu W, Li D, Liu J, Chen S, Qu L, Zheng J, Xu G and Yang N. A genome-wide SNP scan reveals novel loci for egg production and quality traits in white leghorn and brown-egg dwarf layers. PLoS One, 6: e28600. 2011.

Mackay TF, Stone EA and Ayroles JF. The genetics of quantitative traits: challenges and prospects. Nature Reviews Genetics, 10: 565-577. 2009.

Manly KF, Cudmore RHJr and Meer JM. Map Manager QTX, crossplatform software for genetic mapping. Mammalian Genome, 12: 930-932. 2001.
Nadaf J, Pitel F, Gilbert H, Duclos MJ, Vignoles F, Beaumont C, Vignal A, Porter TE, Cogburn LA, Aggrey SE, Simon J and Le Bihan-Duval E. QTL for several metabolic traits map to loci controlling growth and body composition in an $\mathrm{F}_{2}$ intercross between high- and low-growth chicken lines. Physiological Genomics, 38: 241-249. 2009.

Nys Y, Gautron J, Garcia-Ruiz JM and Hincke MT. Avian eggshell mineralization: biochemical and functional characterization of matrix proteins. Comptes Rendus Palevol, 3: 549-562. 2004.

Odabasi AZ, Miles RD, Balaban MO and Portier KM. Changes in brown eggshell color as the hen ages. Poultry Science, 86: 356-363. 2007.

Osman SAM, Sekino M, Nishihata A, Kobayashi Y, Takenaka W, Kinoshita K, Kuwayama T, Nishibori M, Yamamoto Y and Tsudzuki M. The genetic variability and relationships of Japanese and foreign chickens assessed by microsatellite DNA profiling. Asian-Australasian Journal of Animal Sciences, 19: 1369-1378. 2006.

Rieseberg LH, Archer MA and Wayne RK. Transgressive segregation, adaptation and speciation. Heredity, 83: 363-372. 1999.

Roberts JR. Factors affecting egg internal quality and egg shell quality in laying hens. Journal of Poultry Science, 41: 161177. 2004.

Roland DASr. Factors influencing shell quality of aging hens. Poultry Science, 58: 774-777. 1979.

Sasaki O, Odawara S, Takahashi H, Nirasawa K, Oyamada Y, Yamamoto R, Ishii K, Nagamine Y, Takeda H, Kobayashi E and Furukawa T. Genetic mapping of quantitative trait loci affecting body weight, egg character and egg production in F2 intercross chickens. Animal Genetics, 35: 188-194. 2004.

Schmid M, Nanda I, Hoehn H, Schartl M, Haaf T, Buerstedde JM, Arakawa H, Caldwell RB, Weigend S, Burt DW, Smith J, Griffin DK, Masabanda JS, Groenen MAM, Crooijmans RPMA, Vignal A, Fillon V, Morisson M, Pitel F, Vignoles M, Garrigues A, Gellin J, Rodionov AV, Galkina SA, Lukina NA, Ben-Ari G, Blum S, Hillel J, Twito T, Lavi U, David L, Feldman MW, Delany ME, Conley CA, Fowler VM, Hedges SB, Godbout R, Katyal S, Smith C, Hudson Q, Sinclair A and Mizuno S. Second report on chicken genes and chromosomes 2005. Cytogenetic and Genome Research, 109: 415-479. 2005.

Schreiweis MA, Hester PY, Settar P and Moody DE. Identification of quantitative trait loci associated with egg quality, egg production, and body weight in an $\mathrm{F}_{2}$ resource population of chickens. Animal Genetics, 37: 106-112. 2006.

Tadano R, Sekino M, Nishibori M and Tsudzuki M. Microsatellite marker analysis for the genetic relationships among Japanese long-tailed chicken breeds. Poultry Science, 86: 460-469. 2007.

Takahashi H, Tsudzuki M, Sasaki O, Niikura J, Inoue-Murayama M and Minezawa M. A chicken linkage map based on microsatellite markers genotyped on a Japanese Large Game and White Leghorn cross. Animal Genetics, 36: 463-467. 2005.

Tsudzuki M. Japanese native chickens. Pages $91-116$ in The Relationship Between Indigenous Animals and Humans in APEC Region. Chang HL and Huang YC, ed. CSAS, Tainan. 2003.

Tsudzuki M, Onitsuka S, Akiyama R, Iwamizu M, Goto N, Nishibori $\mathrm{M}$, Takahashi $\mathrm{H}$ and Ishikawa A. Identification of quantitative trait loci affecting shank length, body weight and carcass weight from the Japanese cockfighting chicken breed, Oh-Shamo (Japanese Large Game). Cytogenetic and Genome Research, 117: 288-295. 2007. 
Tuiskula-Haavisto M, Honkatukia M, Preisinger R, Schmutz M, de Koning DJ, Wei WH, and Vilkki J. Quantitative trait loci affecting eggshell traits in an $F_{2}$ population. Animal Genetics, 42: 293-299. 2011.

Tuiskula-Haavisto M, Honkatukia M, Vilkki J, de Koning DJ, Schulman NF and Maki-Tanila A. Mapping of quantitative trait loci affecting quality and production traits in egg layers. Poultry Science, 81: 919-927. 2002.

Wahlberg P, Carlborg O, Foglio M, Tordoir X, Syvänen AC, Lathrop M, Gut IG, Siegel PB and Andersson L. Genetic analysis of an F2 intercross between two chicken lines divergently selected for body-weight. BMC Genomics, 10: 248. 2009.

Yao JF, Chen ZX, Xu GY, Wang XL, Ning ZH, Zheng JX, Qu LJ and Yang N. Low-density lipoprotein receptor-related protein 8 gene association with egg traits in dwarf chickens. Poultry Science, 89: 883-886. 2010.

Zhang LC, Ning ZH, Xu GY, Hou ZC and Yang N. Heritabilities and genetic and phenotypic correlations of egg quality traits in brown-egg dwarf layers. Poultry Science, 84: 1209-1213. 2005.

Zhou H, Deeb N, Evock-Clover CM, Ashwell CM and Lamont SJ. Genome-wide linkage analysis to identify chromosomal regions affecting phenotypic traits in the chicken. I. Growth and average daily gain. Poultry Science, 85: 1700-1711. 2006.

Zhou H, Deeb N, Evock-Clover CM, Mitchell AD, Ashwell CM and Lamont SJ. Genome-wide linkage analysis to identify chromosomal regions affecting phenotypic traits in the chicken. III. Skeletal integrity. Poultry Science, 86: 255-266. 2007. 